Article

\title{
An Improved Mesocotyl Elongation Assay for the Rapid Identification and Characterization of Strigolactone-Related Rice Mutants
}

\author{
Suyash Patil ${ }^{\mathbb{D}}$, Syed Adeel Zafar ${ }^{\mathbb{D}}$, Muhammad Uzair, Jinfeng Zhao, Jingjing Fang and \\ Xueyong Li *(i) \\ National Key Facility for Crop Gene Resources and Genetic Improvement, Institute of Crop Science, \\ Chinese Academy of Agricultural Sciences, Beijing, 100081, China; suyashpatil7@gmail.com (S.P.); \\ adeelzafarpbg@gmail.com (S.A.Z.); uzairbreeder@gmail.com (M.U.); zhaojinfeng@caas.cn (J.Z.); \\ fangjingjing@caas.cn (J.F.) \\ * Correspondence: lixueyong@caas.cn; Tel.: +86-10-82107409
}

Received: 20 March 2019; Accepted: 23 April 2019; Published: 25 April 2019 updates

\begin{abstract}
Strigolactones (SLs) constitute an important class of plant hormones involved in diverse developmental activities in plant growth and host-parasite interaction. Although substantial progress has been made to understand this pathway, the mechanism of action is still elusive especially with its interaction with other phytohormones and downstream targets. Here we have utilized the negative role of strigolactones in rice (Oryza sativa L.) mesocotyl elongation as a morphological marker for the identification and characterization of new developmental mutants. We observed that deep sown seeds develop longer mesocotyl compared with the surface-grown seeds in the dark condition. Based on this observation, we have developed a method to access mesocotyl elongation consisting of the glass vessel and vermiculite as a growth media. Mesocotyl elongation in the modified deep sown system results in a many-fold increase compared to the surface-grown seeds in the dark condition. External application of SLs analog rac-GR24 rescued the elongated mesocotyl phenotype in the mutant defective in SLs synthesis but not the signaling mutant, demonstrating its applicability in the physiological experiments. The modified mesocotyl elongation assay can be used as a rapid method for characterization and identification of suppressors/enhancers and new developmental mutants in the SLs or its associated pathway saving a huge amount of time and space.
\end{abstract}

Keywords: mesocotyl; strigolactones; mutagenesis; deep sowing; rice

\section{Introduction}

Strigolactones (SLs) constitute an important class of phytohormones which are involved in diverse biological processes in the plant development and host-parasite interaction. SLs were originally known for their capacity to promote the germination of parasitic plants of the Orobanchaceae family [1]. The presence of SLs in the host and non-host species as a detrimental signal presents an elusive question to the biologists [1,2]. The answers to these questions come from the discovery of the role of SLs in the other biological processes, such as shoot development [3,4], and as a potent hyphal branching factor for arbuscular mycorrhizal fungi [5]. Recently SLs are found to be involved in diverse developmental processes, such as leaf senescence [6,7], root development [8], lamina joint inclination [9], nodulation [10], chilling tolerance [11], drought, salinity stress tolerance [12], nitrate, phosphorous utilization [13,14], and mesocotyl elongation [15]. The identification of high-tillering dwarf mutants in rice ( $d$,dwarf; or $h t d$, high-tillering and dwarf mutants), Arabidopsis (max, more axillary growth), petunia (dad, decreased apical dominance) and pea (rms, ramosus) has made substantial progress 
in elucidating the key players in this pathway [16]. Still, there are many facts yet to be known from this interesting and mysterious signaling pathway and further genetic materials and mutants are needed to be developed.

In this study, we used the recent update on the role of SLs in mesocotyl development to identify and characterize SLs related mutants in rice. Mesocotyl is a specialized organ located between the coleoptilar node and the basal part of the seminal root. Mesocotyl elongation is an important adaptive trait especially in the context of deep sowing tolerance or in the direct-seeding condition [17]. According to previous studies, this trait is regulated by the complex interaction of phytohormones [15,18-21]. SLs restrict mesocotyl elongation in darkness by restricting cell division [15]. The reported methods for assessment of mesocotyl elongation consist of growing the seeds on the surface of solidified agar medium, water culture, sand culture, and paper towel method under constant darkness [22-26]. We noticed that the surface-grown seeds result in a very minute mesocotyl elongation which is very difficult to record without a microscopic setup. Also, the time required for seed processing and maintaining the sterile environment throughout the experiment is a time and resource intensive task.

Here we present an easy and sophisticated method to record mesocotyl length and its possible applications in genetic studies. This method consists of growing the rice seeds at the bottom of the glass vessel filled with vermiculite growth media. The resulting mesocotyl elongation in this method is recorded with a many-fold increase compared with the surface-grown seeds on solidified agar media. Based on this method, we are proposing a lab-based (rather than field-grown) selection procedure to identify and recover rice mutants related to SLs pathway involving different mutagenesis systems, such as Ethyl Methanesulfonate (EMS), gamma irradiation, and transposon or T-DNA insertion populations. Although other developmental mutants may escape, this system can be used for mutation programs mostly focused on SLs related mutants or suppressors/enhancers of known SLs mutants.

\section{Materials and Methods}

\subsection{Plant Materials}

The SLs biosynthesis mutants $d 17, d 10$, and $d 27$ and signaling mutants $d 3$ and $d 14$ were used. Details of these mutants and their corresponding wild type lines are described in Table 1.

Table 1. Details of the mutants and their corresponding wild type lines used in this study.

\begin{tabular}{cccc}
\hline Gene Name & Wild Type & Mutant Name & Type of Mutation \\
\hline$D 10$ & Shiokari & GSOR300181 & L112P [27] \\
$D 17$ & Shiokari & GSOR300184 & Frame-shift deletion [4] \\
$D 27$ & Huaidao 5 & HD118 & L97P \\
$D 3$ & Nipponbare & S2-215 & Q393Stop \\
$D 14$ & Shengdao 16 & SD33 & G296D \\
\hline
\end{tabular}

\subsection{Determination of Effect of Sowing Depth on Mesocotyl Elongation}

Fifteen healthy seeds were sown in the vermiculite media at different sowing depths of $0 \mathrm{~cm}$, $5 \mathrm{~cm}$ and $10 \mathrm{~cm}$ using the glass vessel (diameter of $6 \mathrm{~cm}$; height of $18 \mathrm{~cm}$ ). The vessels were watered and kept in the cardboard box covered with a black cloth in a $28^{\circ} \mathrm{C}$ growth chamber with constant darkness for 10 days.

\subsection{Growing the Seeds on the Surface of Solidified Agar Media}

The well dried de-husked seeds of rice were sterilized with $20 \%$ sodium hypo-chloride solution (V/V) for 45 minutes with constant shaking. The seeds were then washed thoroughly with sterile de-ionized water for at least five times. The seeds were then planted on the surface of the hydroponic media solidified with $0.6 \%$ agar in the glass bottle. The glass bottles were then placed in the cardboard 
boxes covered with black cloth [15]. The boxes were then kept at $28^{\circ} \mathrm{C}$ growth chamber with constant darkness for 10 days.

\subsection{Growing the Seeds in the Modified System Using Vermiculite Media}

In the proposed modified system, well dried, unprocessed seeds were used. First, the glass vessel (diameter of $6 \mathrm{~cm}$; height of $18 \mathrm{~cm}$ ) is filled with $5 \mathrm{~cm}$ layer of vermiculite media and sprinkled with 15 healthy seeds. The number can be increased by up to 20 seeds per bottle depending on the experimental needs. Then the remaining portion is filled with vermiculite growth media making an overlay of $10 \mathrm{~cm}$ leaving a $3 \mathrm{~cm}$ space. The bottles were then supplemented with $200 \mathrm{~mL}$ tap water having adjusted $\mathrm{pH}$ value of 5.8. The glass vessels were then placed in the cardboard boxes covered with black cloth. The boxes were then kept at $28^{\circ} \mathrm{C}$ in a growth chamber with constant darkness for 10 days.

\subsection{SLs Application in the Modified System}

The synthetic SL analog rac-GR24 was purchased from Chiralix (product number: CX23880) and $10 \mathrm{mM}$ stock solution was prepared using $100 \%$ acetone as a solvent. The glass vessels already sown with the seeds and filled with the vermiculite media was supplemented with $200 \mathrm{~mL}$ tap water containing $0,0.01,0.1$, and $1.0 \mu \mathrm{M}$ GR2 4 as a final concentration. A mock treatment containing an equivalent amount of acetone is also used as a negative control. The bottles were then kept for 10 days in the grown chambers as described in the general method.

\subsection{Data Recording and Statistical Analysis}

The mesocotyl length (distance between the points of seminal root emergence and the coleoptilar node) was recorded using a ruler for individual seedlings. The average of 10 uniformly grown seedlings was represented for each treatment. One-way ANOVA with Tukey's multiple comparison post-hoc test was performed using GraphPad Prism version 5.00 for Windows, GraphPad Software, San Diego, CA, USA.

\section{Results}

\subsection{The Modified Mesocotyl Elongation System}

Mesocotyl elongation is an important trait in the context of deep sowing tolerance and its complex interaction with phytohormones. We noticed that deep sown seedlings develop longer mesocotyl compared with the surface-grown seeds in the dark condition. We performed an experiment growing seeds of the SLs-deficient $d 27$ mutant with its corresponding wild type line at different sowing depths of $0 \mathrm{~cm}, 5 \mathrm{~cm}$, and $10 \mathrm{~cm}$ under the condition of complete darkness. The results suggest that mesocotyl length increases with increasing the sowing depth and reaches its maximum level at $10 \mathrm{~cm}$ depth (Figure 1A,B). Based on this observation, we developed a system using glass vessels of $18 \mathrm{~cm}$ long and $6 \mathrm{~cm}$ diameter using vermiculite as a growth media (Figure 2). Vermiculite provides enough aeration for the developing seedlings due to its porous nature and holds enough moisture to be able to supplement for 10 days. Also, seedling collection and cleaning becomes very easy at the end of the experiment. An additional movie file illustrating the method of seedling collection in the proposed modified mesocotyl elongation method is shown in detail (see Supplementary Video S1). This provides an excellent opportunity to record the observations very quickly without any microscopic setup. This suggests that this system can be effectively used for experiments involving a large number of populations, such as genome-wide association study (GWAS) or QTL mapping. 


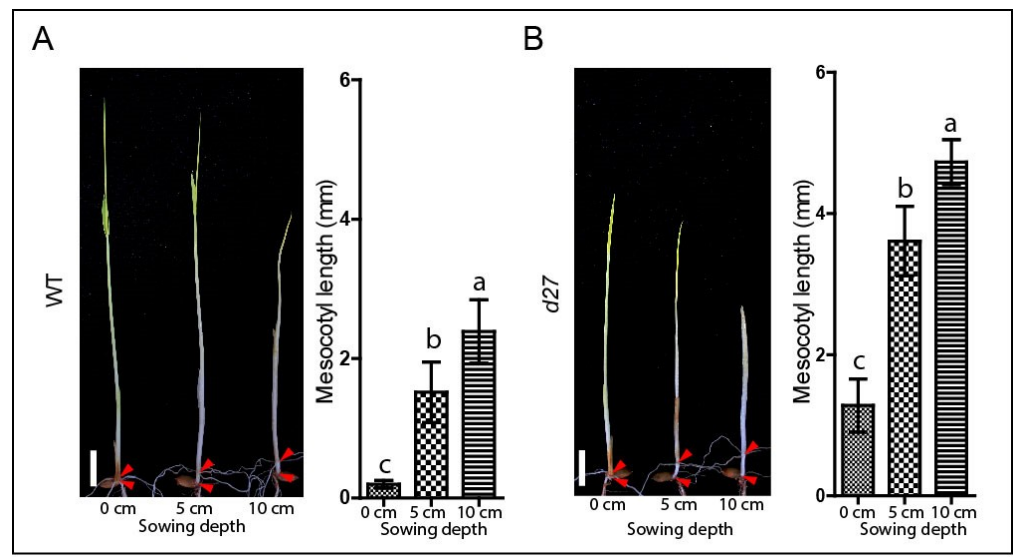

Figure 1. Sowing depth enhances mesocotyl elongation in rice grown for 10 days in the dark. (A) Effect of different sowing depths on the WT line. (B) Effect of different sowing depths on the $d 27$ mutant. Upper and lower arrowhead delimit the mesocotyl. Scale bar $=1 \mathrm{~cm}$. Different lower case letters denote significant differences ( $p<0.05$, one-way ANOVA following Tukey's test for multiple comparisons). Error bars represents $\pm \mathrm{SD}(n=10)$.

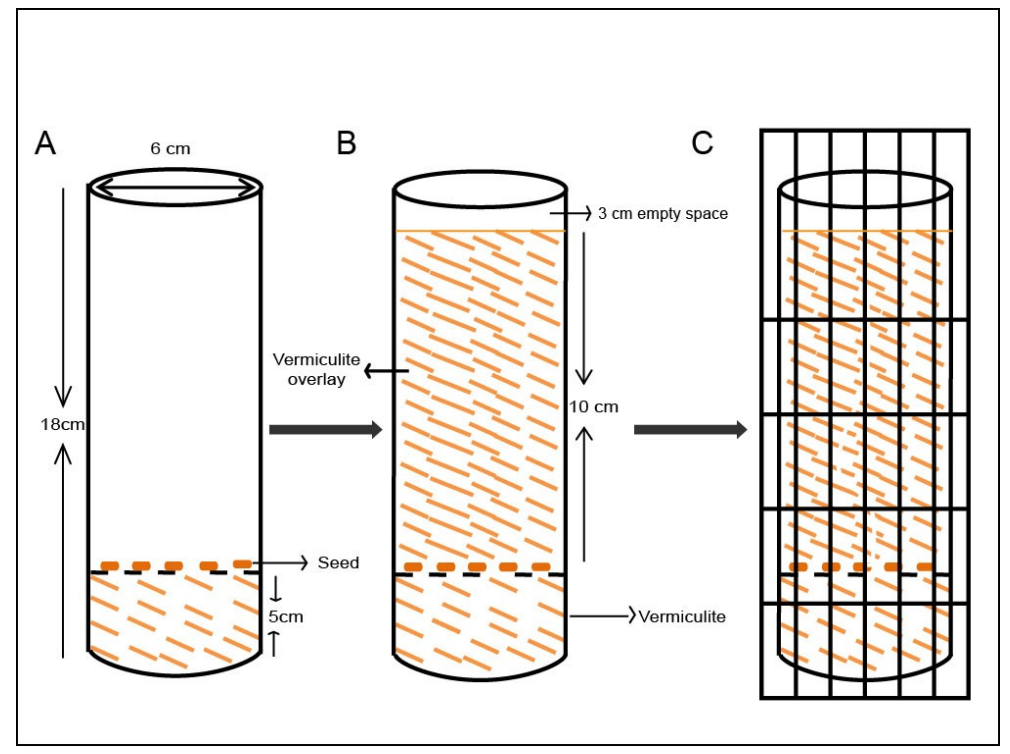

Figure 2. Graphical representation of the improved mesocotyl elongation assay. (A) The initial process of filling the glass vessel with vermiculite and sowing the seeds. (B) The process of filling the remaining portion of the glass vessel with vermiculite media and application of treatments. (C) Placing the glass vessels in the dark place. Grid lines represent cardboard boxes covered with black cloth.

\subsection{SLs-Related Mutants Exhibit Longer Mesocotyl Length in the Modified System}

Rice loss-of-function mutants in the SLs biosynthesis (d10, d17, and d27) and signaling ( $d 3$ and d14) pathway were used (Table 1). To compare the effect of surface and deep sowing condition on mesocotyl elongation, the seeds were either grown on the surface of solidified agar media or in the modified deep sowing condition. The results suggest that in both systems, all the mutants exhibited longer mesocotyl organ compared with the wild type lines (Figure 3). Earlier reports also suggest that all the SLs pathway mutants exhibit longer mesocotyl organ compared with the wild type line under the condition of darkness [15]. However, the resulting elongation in both wild type and mutant lines were difficult to judge with naked eyes and required microscopic setup for surface sown treatment (Figure 3A). Interestingly, deep sown seeds developed a longer mesocotyl organ compared to the surface sown seeds. In almost all the lines, the mesocotyl length was recorded with a many-fold increase in the 
modified system compared with the surface-grown seeds. The $d 10$ mutant exhibited a maximum fold increase ( 10 fold) followed by $d 3$ ( 5 fold) in the deep sown condition compared with the surface-grown seedlings (Figure 3C,D). The elongation was easy to measure using a ruler and did not require a microscopic setup. In both the systems, the $d 3$ mutant exhibited maximum mesocotyl elongation (Figure 3C,D). The different wild type lines also recorded with differential mesocotyl elongation rates which suggest the influence of genetic makeup on this trait (Table 1).

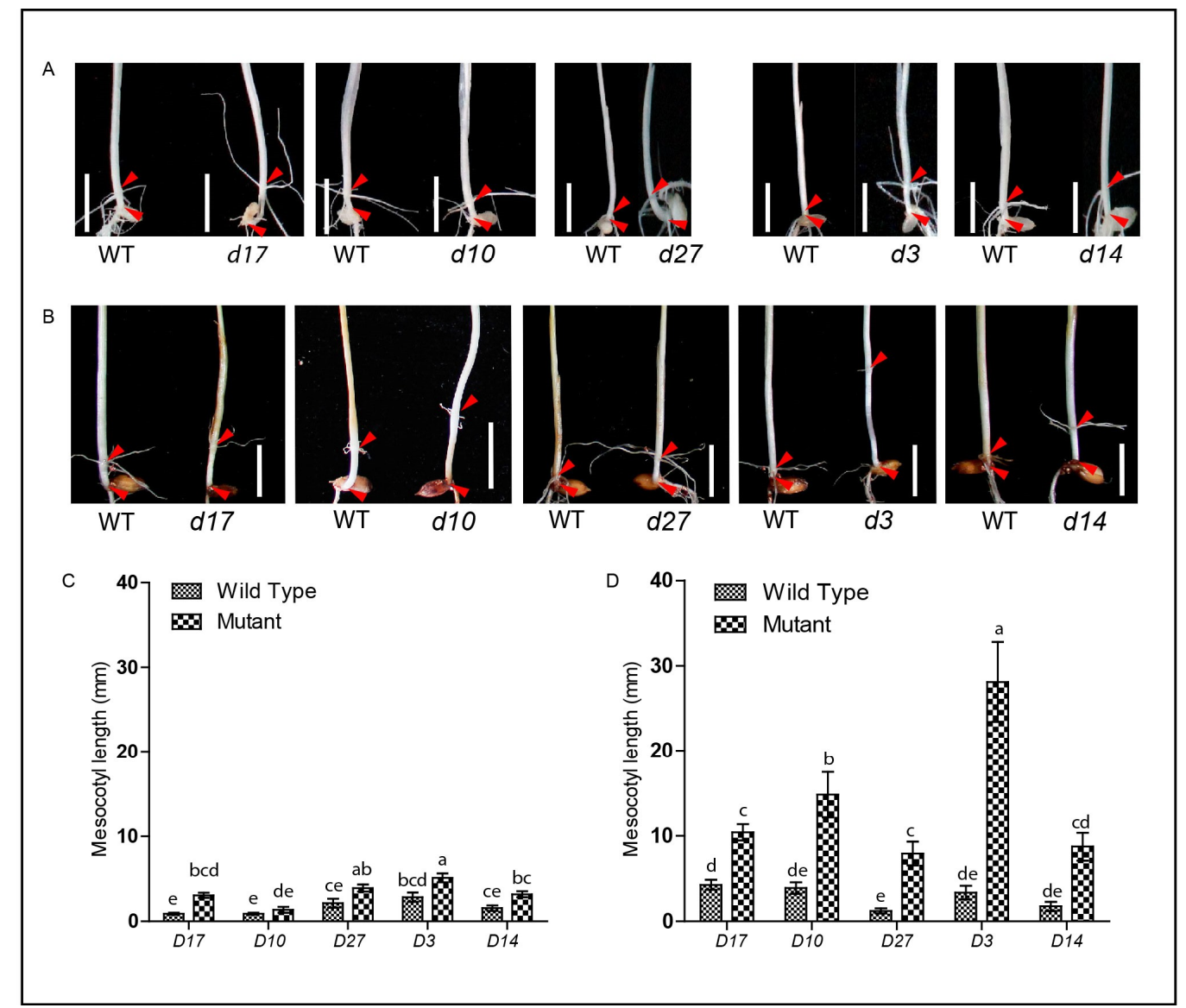

Figure 3. Comparison of the mesocotyl elongation following surface-sown or deep sown modified system. (A) Mesocotyl elongation of wild type (left) and the $d$ mutants (right) grown on the surface of solidified agar medium for 10 days. (B) Mesocotyl elongation of wild type (left) and the $d$ mutants (right) grown with the modified deep sown method for 10 days. Upper arrowhead indicates the position of the coleoptiler node and the lower arrowhead represents the position of the basal part of the seminal root. Mesocotyl is the tissue between the upper and lower arrowhead. Bars $=1 \mathrm{~cm}$. (C) Mesocotyl length of 10-day-old seedlings grown under constant darkness following a surface grown method. Values represents mean \pm SD $(n=10)$. (D) Mesocotyl length of 10 days old seedlings grown under constant darkness following a modified deep sowing method. Different lower case letters denote significant differences ( $p<0.05$, one-way ANOVA following Tukey's test for multiple comparisons).

Values represents mean $\pm \mathrm{SD}(n=10)$.

\subsection{GR24 Rescues the Elongated Mesocotyl in SL-Deficient Mutant but Not in the Signaling Mutant Following the Modified System}

As a known phenomenon, the exogenous application of synthetic SLs analog GR24 can rescue the elongated mesocotyl phenotype in the SLs deficient mutants but not in the signaling mutants $[15,18,21]$. We tested this using the modified system to check its effectiveness using the SLs biosynthesis mutant $d 27$ and signaling mutant $d 14$. Using the modified system, the exogenous application of GR24 completely rescued the elongated mesocotyl phenotype in the SLs biosynthesis mutant $d 27$ (Figure 4A,C) but not 
in the signaling mutant $d 14$ (Figure $4 \mathrm{~B}, \mathrm{D}$ ). A concentration of $0.1 \mu \mathrm{M}$ is required for complete rescue of the $d 27$ phenotype to wild type level (Figure $4 \mathrm{~A}$ ). This suggests that the improved system can be used for initial characterization of newly identified high-tillering dwarf mutants to classify into the signaling and biosynthesis components. If the application of GR24 rescues the phenotype, this can narrow down the search towards biosynthesis components.

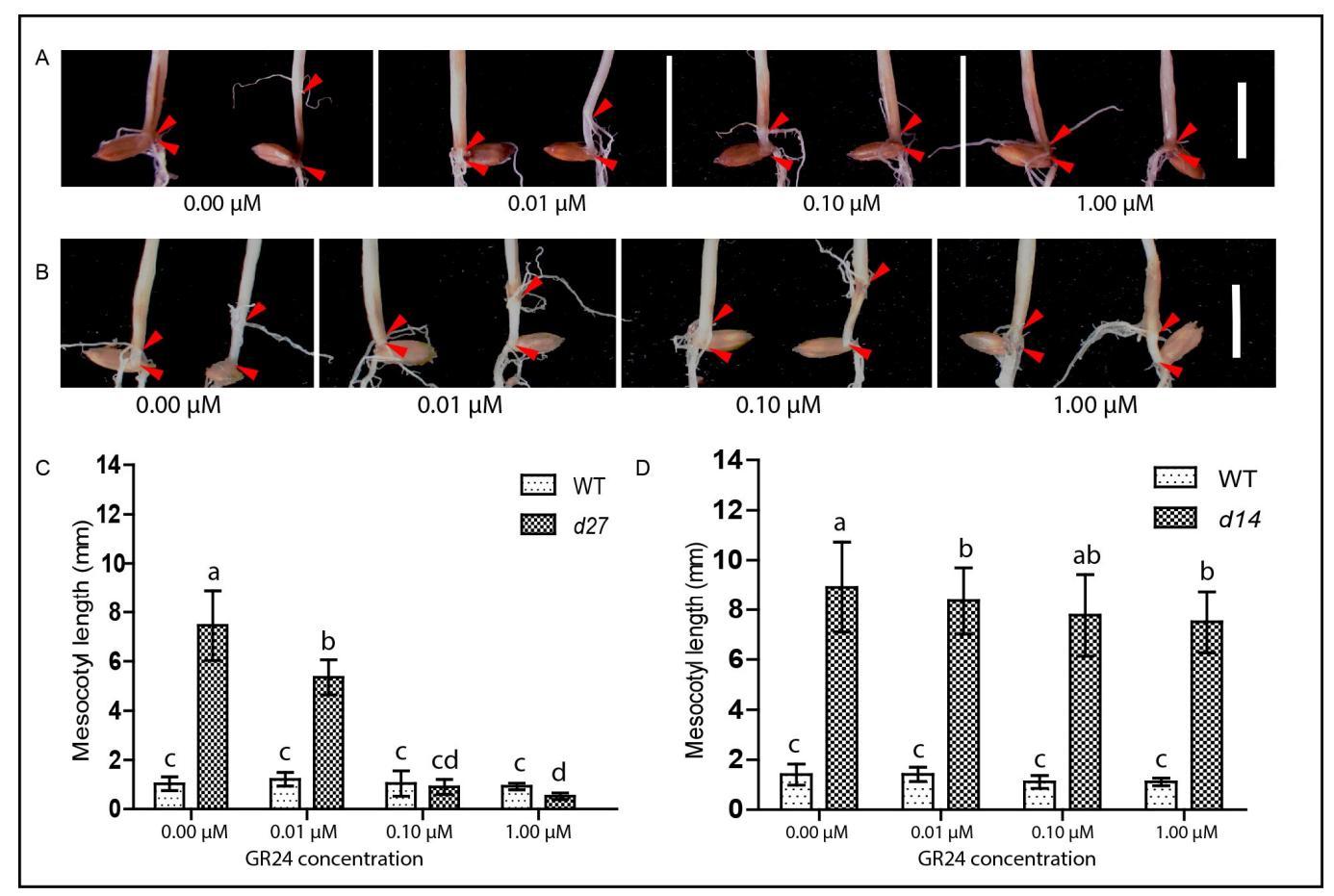

Figure 4. Effect of GR24 on the mesocotyl elongation of WT and the $d$ mutants. (A) Effect of external application of GR24 on mesocotyl elongation of wild type (left) and the $d 27$ mutant (right) grown with a modified method for 10 days in the dark. (B) Effect of external application of GR24 on mesocotyl elongation of wild type (left) and the $d 14$ mutant (right) grown with a modified method for 10 days in the dark. Upper arrowhead indicates the position of the coleoptiler node and the lower arrowhead represents the position of the basal part of the seminal root. Mesocotyl is the tissue between the upper and lower arrowhead. Bars $=1 \mathrm{~cm}$ (C) Effect of external application of GR24 on mesocotyl length of WT and the $d 27$ mutant following the modified deep sowing method. Values represents mean \pm SD $(n=10)$. (D) Effect of external application of GR24 on mesocotyl length of WT and the $d 14$ mutant following the modified deep sowing method. Values represents mean $\pm \operatorname{SD}(n=10)$. Different lower case letters denote significant differences ( $p<0.05$, one-way ANOVA following Tukey's test for multiple comparisons).

\section{Discussion}

Here we present an easy and rapid method for the assessment of mesocotyl elongation with its possible application in genetic studies. SLs play an intrinsic role in the regulation of mesocotyl elongation in response to darkness. Many researchers are working on the mesocotyl elongation trait using mutants as well as GWAS and QTL mapping approach $[23,25,26]$. The previous methods described for the assessment of mesocotyl elongation were found to be time and resource intensive which results in minute elongation rates. This makes the data recording and analysis difficult and labor intensive for experiments, such as GWAS or QTL mapping. Keeping this in mind, we utilized an observation which suggests that deep sowing results in an enhancement in mesocotyl elongation (Figure 1). The proposed modified system consists of an easy-to-follow method utilizing vermiculite as an inert media. The mesocotyl elongation in the modified system is recorded with a many-fold increase and this makes the data recording easy and accurate. 
The reason for the enhanced mesocotyl length in the modified system can be explained with the recent report on the ethylene-mediated inhibition of the jasmonic acid biosynthesis [20]. Under the condition of deep sowing, the emerging seedlings make many adjustments to make successful germination. Ethylene is an important mediator of soil overlay response of the germinating seedlings and soil cover quantitatively correlates with ethylene production [28]. Jasmonic acid (JA) restricts cell elongation in the mesocotyls of etiolated rice seedlings, whereas ethylene inhibits JA biosynthesis through down-regulation of the initial JA biosynthetic component GY1 [20]. This suggests that the enhanced mesocotyl elongation in the modified system consisting of $10 \mathrm{~cm}$ overlay of vermiculite media might be the result of enhanced ethylene production. Since jasmonic acid is involved in cell elongation and SLs restricts cell division, these two phytohormones will not interfere with each other's functions (Figure 5).

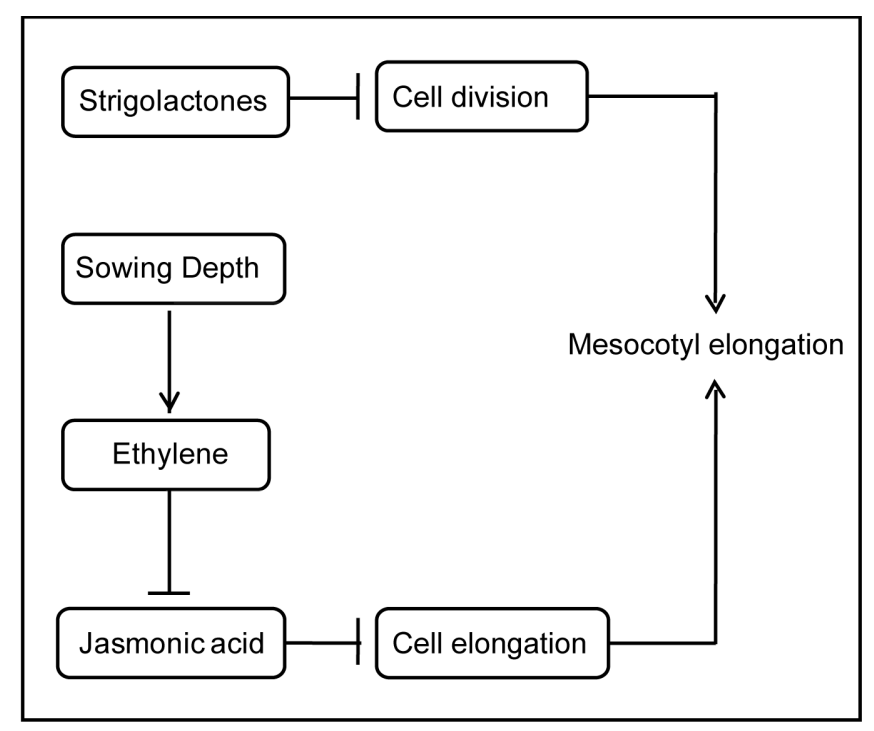

Figure 5. The working model for the possible mechanism of enhanced mesocotyl elongation in the modified deep sowing method. The arrowhead indicates promotion and bar represents restriction.

The external application of SLs analog rac-GR24 rescued the phenotype of strigolactone deficient mutant $d 27$ but not that of the signaling mutant $d 14$ suggesting that the system is suitable for physiological experiments (Figure 4). In rice, the characterization of tiller bud outgrowth of SLs-related mutants in response to GR24 treatment takes at least two weeks which requires repeated changes of the growth media [22,29]. Whereas, the mesocotyl elongation experiment requires only 10 days to assess the phenotype with only one time GR24 application. This suggests the applicability and reliability of this system for functional characterization and classification of the new mutants into biosynthesis and signaling components. SLs are now being used as potent agrochemicals due to its capacity to modulate plant architecture and involvement in symbiosis with arbuscular mycorrhizal fungi and as a suicide germinating agent to kill the parasitic weeds [30]. Despite these benefits, SLs are still far behind for its commercial use due to the complexities in production and lack of an easy system to test newly developed analogs. We propose that this system can be used as an easy and quick method to test newly developed SLs analogs/mimics.

Identification of the new developmental mutants and its underlying cause is a lengthy and resource-intensive task [31]. Space is a major constraint for growing crops and constitutes a major element in the research budget. In most of the rice mutagenesis experiments, at least $5000 \mathrm{M}_{1}$ plants are required in the population. In the $\mathrm{M}_{2}$ generation, 15 plants from each line are required to grow which consumes around 0.5 hectares of land and a huge amount of labor and resources. We hypothesize that a true mutant in the strigolactone related pathway will result in mesocotyl elongation in response to deep sowing in the modified system. In mutagenesis experiments, $\mathrm{M}_{2}$ generation will typically 
segregate for this trait and such lines can be further grown in the field. The progenies showing developmental alterations (such as tiller number and plant height) compared with the wild type line could be selected for further mapping population development. The proposed model utilizes the modified system for assessment of mesocotyl elongation in the mutagenesis population. The tight relation of mesocotyl elongation and defective strigolactone signaling provides an excellent opportunity for selecting mutants impaired in the same or its interacting pathway. Although there are chances of losing other developmental mutants, this system can be used for mutation programs specially focused on SLs-or other interacting phytohormones like cytokinins, brassinosteroids, ethylene, and jasmonic acid related mutants.

\section{Conclusions}

Here we present an easy and robust way to identify and characterize mutants in the SLs pathway. Mesocotyl elongation is used as a morphological marker for disturbed SLs signaling based on the previous reports. Due to some technical difficulties in data recording and handling large populations in the traditional method, we have developed an improved mesocotyl elongation assay. The improved system fits well in handling large populations and does not require a sterile environment and microscopic setup to record the observations. This assay can also be used in the characterization of SLs-related mutants to classify them into biosynthesis or signaling mutants. Finally, the idea of enhanced mesocotyl elongation in the modified system can be used for identification of mutants in the early generation screen and can speed up the mutant recovery process saving a huge amount of time and resources.

Supplementary Materials: The following are available online at http://www.mdpi.com/2073-4395/9/4/208/s1, Video S1: The procedure of seedling collection. The video shows the procedure of seedling collection from the proposed modified mesocotyl elongation method. The seedlings are grown in dark condition for 10 days.

Author Contributions: Conceptualization, S.P. and X.L.; methodology, S.P.; validation, S.P., and X.L.; investigation, S.P., S.A.Z., M.U., J.Z., J.F., and X.L.; writing—original draft preparation, S.P.; writing—review and editing, S.P., and X.L.; supervision, X.L.

Funding: This work was supported by grants from the National Natural Science Foundation of China (31670279 and 31271311 to XL), and the Agricultural Science and Technology Innovation Program of Chinese Academy of Agricultural Sciences.

Acknowledgments: SBP is a Ph.D. scholar supported by the China Scholarship Council (CSC) and Ministry of Human Resource Development, Government of India. We thank Dale Bumpers National Rice Research Center (Stuttgart, AR, USA) for providing the rice seeds of $d 10$ (GSOR300181), d17 (GSOR300184), and Shiokari (GSOR300192). We also thank Shoujiang Yuan at the Shandong Rice Research Institute (Jinan, Shandong, China) for propagating rice seeds.

Conflicts of Interest: The authors declare no conflict of interest.

\section{References}

1. Cook, C.E.; Whichard, L.P.; Turner, B.; Wall, M.E.; Egley, G.H. Germination of Witchweed (Striga lutea Lour.): Isolation and Properties of a Potent Stimulant. Science 1966, 154, 1189-1190. [CrossRef] [PubMed]

2. Siame, B.A.; Weerasuriya, Y.; Wood, K.; Ejeta, G.; Butler, L.G. Isolation of strigol, a germination stimulant for Striga asiatica, from host plants. J. Agric. Food Chem. 1993, 41, 1486-1491. [CrossRef]

3. Gomez-Roldan, V.; Fermas, S.; Brewer, P.B.; Puech-Pages, V.; Dun, E.A.; Pillot, J.P.; Letisse, F.; Matusova, R.; Danoun, S.; Portais, J.C.; et al. Strigolactone inhibition of shoot branching. Nature 2008, 455, 189-194. [CrossRef] [PubMed]

4. Umehara, M.; Hanada, A.; Yoshida, S.; Akiyama, K.; Arite, T.; Takeda-Kamiya, N.; Magome, H.; Kamiya, Y.; Shirasu, K.; Yoneyama, K.; et al. Inhibition of shoot branching by new terpenoid plant hormones. Nature 2008, 455, 195-200. [CrossRef] [PubMed]

5. Akiyama, K.; Matsuzaki, K.; Hayashi, H. Plant sesquiterpenes induce hyphal branching in arbuscular mycorrhizal fungi. Nature 2005, 435, 824-827. [CrossRef] 
6. Ueda, H.; Kusaba, M. Strigolactone Regulates Leaf Senescence in Concert with Ethylene in Arabidopsis. Plant Physiol. 2015, 169, 138-147. [CrossRef]

7. Yamada, Y.; Umehara, M. Possible Roles of Strigolactones during Leaf Senescence. Plants (Basel, Switzerland) 2015, 4, 664-677. [CrossRef]

8. Kapulnik, Y.; Koltai, H. Strigolactone Involvement in Root Development, Response to Abiotic Stress, and Interactions with the Biotic Soil Environment. Plant Physiol. 2014, 166, 560-569. [CrossRef]

9. Li, X.; Sun, S.; Li, C.; Qiao, S.; Wang, T.; Leng, L.; Shen, H.; Wang, X. The Strigolactone-related mutants have enhanced lamina joint inclination phenotype at the seedling stage. J. Genet. Genom. 2014, 41, 605-608. [CrossRef]

10. Foo, E.; Davies, N.W. Strigolactones promote nodulation in pea. Planta 2011, 234, 1073-1081. [CrossRef]

11. Cooper, J.W.; Hu, Y.; Beyyoudh, L.; Yildiz Dasgan, H.; Kunert, K.; Beveridge, C.A.; Foyer, C.H. Strigolactones positively regulate chilling tolerance in pea and in Arabidopsis. Plant Cell Environ. 2018, 41, 1298-1310. [CrossRef]

12. Ha, C.V.; Leyva-Gonzalez, M.A.; Osakabe, Y.; Tran, U.T.; Nishiyama, R.; Watanabe, Y.; Tanaka, M.; Seki, M.; Yamaguchi, S.; Dong, N.V.; et al. Positive regulatory role of strigolactone in plant responses to drought and salt stress. Proc. Natl. Acad. Sci. USA 2014, 111, 851-856. [CrossRef]

13. Mayzlish-Gati, E.; De-Cuyper, C.; Goormachtig, S.; Beeckman, T.; Vuylsteke, M.; Brewer, P.B.; Beveridge, C.A.; Yermiyahu, U.; Kaplan, Y.; Enzer, Y.; et al. Strigolactones are involved in root response to low phosphate conditions in Arabidopsis. Plant Physiol. 2012, 160, 1329-1341. [CrossRef]

14. Sun, H.; Tao, J.; Liu, S.; Huang, S.; Chen, S.; Xie, X.; Yoneyama, K.; Zhang, Y.; Xu, G. Strigolactones are involved in phosphate- and nitrate-deficiency-induced root development and auxin transport in rice. J. Exp. Bot. 2014, 65, 6735-6746. [CrossRef]

15. Hu, Z.; Yan, H.; Yang, J.; Yamaguchi, S.; Maekawa, M.; Takamure, I.; Tsutsumi, N.; Kyozuka, J.; Nakazono, M. Strigolactones negatively regulate mesocotyl elongation in rice during germination and growth in darkness. Plant Cell Physiol. 2010, 51, 1136-1142. [CrossRef]

16. Marzec, M. Perception and Signaling of Strigolactones. Front Plant Sci. 2016, 7, 1260. [CrossRef]

17. Lee, H.S.; Sasaki, K.; Kang, J.W.; Sato, T.; Song, W.Y.; Ahn, S.N. Mesocotyl Elongation is Essential for Seedling Emergence Under Deep-Seeding Condition in Rice. Rice (NY) 2017, 10, 32. [CrossRef]

18. Sun, S.; Wang, T.; Wang, L.; Li, X.; Jia, Y.; Liu, C.; Huang, X.; Xie, W.; Wang, X. Natural selection of a GSK3 determines rice mesocotyl domestication by coordinating strigolactone and brassinosteroid signaling. Nat. Commun. 2018, 9, 2523. [CrossRef]

19. Takahashi, K. Interaction between ethylene, abscisic acid and gibberellic acid in elongation of rice mesocotyl. Planta 1973, 109, 363-364. [CrossRef]

20. Xiong, Q.; Ma, B.; Lu, X.; Huang, Y.H.; He, S.J.; Yang, C.; Yin, C.C.; Zhao, H.; Zhou, Y.; Zhang, W.K.; et al. Ethylene-Inhibited Jasmonic Acid Biosynthesis Promotes Mesocotyl/Coleoptile Elongation of Etiolated Rice Seedlings. Plant Cell 2017, 29, 1053-1072. [CrossRef]

21. Hu, Z.; Yamauchi, T.; Yang, J.; Jikumaru, Y.; Tsuchida-Mayama, T.; Ichikawa, H.; Takamure, I.; Nagamura, Y.; Tsutsumi, N.; Yamaguchi, S.; et al. Strigolactone and cytokinin act antagonistically in regulating rice mesocotyl elongation in darkness. Plant Cell Physiol. 2014, 55, 30-41. [CrossRef]

22. Liu, L.; Xie, T.; Peng, P.; Qiu, H.; Zhao, J.; Fang, J.; Patil, S.B.; Wang, Y.; Fang, S.; Chu, J.; et al. Mutations in the MIT3 gene encoding a caroteniod isomerase lead to increased tiller number in rice. Plant Sci. 2018, 267, 1-10. [CrossRef]

23. Lu, Q.; Zhang, M.; Niu, X.; Wang, C.; Xu, Q.; Feng, Y.; Wang, S.; Yuan, X.; Yu, H.; Wang, Y.; et al. Uncovering novel loci for mesocotyl elongation and shoot length in indica rice through genome-wide association mapping. Planta 2016, 243, 645-657. [CrossRef]

24. Tamiru, M.; Abe, A.; Utsushi, H.; Yoshida, K.; Takagi, H.; Fujisaki, K.; Undan, J.R.; Rakshit, S.; Takaichi, S.; Jikumaru, Y.; et al. The tillering phenotype of the rice plastid terminal oxidase (PTOX) loss-of-function mutant is associated with strigolactone deficiency. New Phytol. 2014, 202, 116-131. [CrossRef]

25. Wu, J.; Feng, F.; Lian, X.; Teng, X.; Wei, H.; Yu, H.; Xie, W.; Yan, M.; Fan, P.; Li, Y.; et al. Genome-wide Association Study (GWAS) of mesocotyl elongation based on re-sequencing approach in rice. BMC Plant Biol. 2015, 15, 218. [CrossRef] 
26. Zhao, Y.; Zhao, W.; Jiang, C.; Wang, X.; Xiong, H.; Todorovska, E.G.; Yin, Z.; Chen, Y.; Wang, X.; Xie, J.; et al. Genetic Architecture and Candidate Genes for Deep-Sowing Tolerance in Rice Revealed by Non-syn GWAS. Front Plant Sci. 2018, 9, 332. [CrossRef]

27. Arite, T.; Iwata, H.; Ohshima, K.; Maekawa, M.; Nakajima, M.; Kojima, M.; Sakakibara, H.; Kyozuka, J. DWARF10, an RMS1/MAX4/DAD1 ortholog, controls lateral bud outgrowth in rice. Plant J. 2007, 51, 1019-1029. [CrossRef]

28. Zhong, S.; Shi, H.; Xue, C.; Wei, N.; Guo, H.; Deng, X.W. Ethylene-orchestrated circuitry coordinates a seedling's response to soil cover and etiolated growth. Proc. Natl. Acad. Sci. USA 2014, 111, 3913-3920. [CrossRef]

29. Zhao, J.; Wang, T.; Wang, M.; Liu, Y.; Yuan, S.; Gao, Y.; Yin, L.; Sun, W.; Peng, L.; Zhang, W.; et al. DWARF3 participates in an SCF complex and associates with DWARF14 to suppress rice shoot branching. Plant Cell Physiol. 2014, 55, 1096-1109. [CrossRef]

30. Vurro, M.; Prandi, C.; Baroccio, F. Strigolactones: How far is their commercial use for agricultural purposes? Pest Manag. Sci. 2016, 72, 2026-2034. [CrossRef]

31. Garcia, V.; Bres, C. Rapid identification of causal mutations in tomato EMS populations via mapping-by-sequencing. Nat. Protoc. 2016, 11, 2401-2418. [CrossRef]

(C) 2019 by the authors. Licensee MDPI, Basel, Switzerland. This article is an open access article distributed under the terms and conditions of the Creative Commons Attribution (CC BY) license (http://creativecommons.org/licenses/by/4.0/). 